\title{
BEST PRACTICE
}

Managing liver failure

\section{A Kelly}

Liver disease is rare in childhood, but important new developments have altered the natural history and outcome. It is important that clinicians are aware of these diseases and their management. Acute liver failure is most often due to viral hepatitis, paracetamol overdose, or inherited metabolic liver disease. The clinical presentation includes jaundice, coagulopathy, and encephalopathy. Early diagnosis is necessary to prevent complications such as cerebral oedema, gastrointestinal bleeding, and renal failure. Early supportive management, in particular intravenous $\mathrm{N}$-acetylcysteine, may be effective but liver transplantation is usually the definitive treatment and thus early referral to a specialist unit for liver transplantation is mandatory. Chronic liver failure may be due to unresolved neonatal liver disease, either inherited biliary hypoplasia or extrahepatic biliary atresia, while in older children, autoimmune liver disease or cystic fibrosis are the commonest causes. Treatment includes specific medication, nutritional support, and liver transplantation, which now has a $90 \%$ survival with good quality life.

Correspondence to: Professor Deirdre A Kelly, Liver Unit, Birmingham Children's Hospital NHS Trust, Steelhouse Lane, Birmingham B4 6NH, UK Deirdre.Kelly@ bhamchildrens.wmids.nhs.uk

Submitted 27 May 2002 Accepted 19 June 2002

\section{ACUTE LIVER FAILURE}

Acute liver failure or fulminant hepatitis is rare in childhood, but has a mortality of $70 \%$ without appropriate management and/or liver transplantation. Fulminant hepatic failure is defined as encephalopathy within eight weeks of the onset of liver disease. This definition is not useful for paediatric acute liver failure as the clinical presentation may be prolonged particularly if secondary to autoimmune or metabolic liver disease. ${ }^{1}$

The acute liver damage may be secondary to a virus, drug, or toxin or immune mediated attack. The underlying mechanism is unknown but is multifactorial and depends on the age, susceptibility of the patient, and the extent of hepatic injury. The main pathological features include severe hepatic necrosis, loss of hepatic architecture, and absence of hepatic regeneration.

The aetiology of acute liver failure varies depending on the age of the child (box 1). In neonates, inherited metabolic liver disease or infection is likely while viral hepatitis, autoimmune liver disease, or drug induced liver failure are common in older children. the development of hepatic necrosis with

\section{Clinical presentation}

The clinical presentation depends on the aetiology of acute liver failure but the presentation may either be acute, within days, or prolonged to 10 weeks if due to metabolic liver disease. The extent of jaundice and encephalopathy is variable in the early stages, but all children have coagulopathy. Encephalopathy is particularly difficult to diagnose in neonates. Vomiting and poor feeding are early signs, while irritability and reversal of day/night sleep patterns indicates more established hepatic encephalopathy. In older children encephalopathy may present with aggressive behaviour or convulsions.

\section{Neonatal acute liver failure}

The commonest cause of acute liver failure in neonates is septicaemia secondary to Escherichia coli, Staphylococcus aureus, or herpes simplex (box 1 and table 1). Other causes include adenovirus, echovirus, and Coxsackie virus. Acute liver failure secondary to hepatitis B usually presents at about 12 weeks of age while hepatitis A is rare in neonates. Hepatitis $\mathrm{C}$ does not cause acute liver failure in neonates.

\section{Hepatitis B}

Hepatitis B is vertically transmitted during pregnancy or delivery. The transmission rate is approximately $70 \%$ in mothers who are hepatitis B surface antigen and hepatitis B e antigen positive. Most infected infants will become asymptomatic carriers. In contrast, infants born to mothers who are hepatitis B e antigen negative have a high risk of developing fulminant hepatitis within the first 12 weeks of life due the transmission of a pre-core mutant hepatitis virus from mother to child..$^{23}$ Both the development of the carrier state and fulminant hepatitis B may be prevented by vaccination of all infants of hepatitis B carrier mothers, irrespective of their e antigen status.

\section{Neonatal haemochromatosis}

Neonatal haemochromatosis is a rare disorder, which is associated with iron accumulation in liver, pancreas, heart, and brain. It is not known whether the disease is inherited with autosomal recessive inheritance or is an acquired defect of iron handling in pregnancy. Clinical presentation may be within hours or weeks of birth with jaundice, hypoglycaemia, and severe coagulopathy. Encephalopathy, although present may not be

Abbreviations: FIC, familial intrahepatic cholestasis; NTBC, 2 (2 nitro-4-trifluoromethylbenzoyl)-1, 3-cyclohexenedione; TIPSS, transjugular intrahepatic portosystemic stent shunt 


\section{Box 1: Aetiology of acute liver failure}

\section{Neonate/infant up to 6 months}

- Infection: septicaemia, hepatitis B, adenovirus, echovirus, Coxsackie B.

- Metabolic: neonatal haemochromatosis, tyrosinaemia type I, mitochondrial disorders, fatty acid oxidation defects.

- Poisoning: paracetamol.

- Familial haemophagocytic syndrome.

\section{Children $>6$ months}

- Viral hepatitis: hepatitis $A / B / E / n o n-A-G$, Epstein-Barr virus, parvovirus B19.

- Autoimmune type I or II.

- Drug induced: paracetamol overdose, sodium valproate, carbamazepine, isoniazid, halothane.

- Metabolic: Wilson's disease, Alpers disease.

obvious. The diagnosis is suggested by raised ferritin levels (2000-3000 $\mu \mathrm{g} / \mathrm{l})$ and confirmed by demonstrating a high serum iron with hypersaturation of iron binding capacity $(95 \%-105 \%)$ and the demonstration of extrahepatic haemosiderosis. Because of the severe coagulopathy liver biopsy is usually contraindicated but extrahepatic siderosis may be demonstrated in salivary glands obtained by lip biopsy or the demonstration of iron accumulation in pancreas and brain on magnetic resonance imaging. Intensive supportive management of the liver failure and an antioxidant cocktail (boxes 2 and 3) may be effective if begun within 24-48 hours of birth in milder cases but liver transplantation is usually required. ${ }^{4}$

\section{Tyrosinaemia type I}

Tyrosinaemia type $\mathrm{I}$ is an autosomal recessive disorder secondary to deficiency of fumaryl acetoacetase which prevents the metabolism of tyrosine leading to the accumulation of toxic metabolites, which damage liver, kidneys, heart, and brain. Infants present between 1 and 6 months of age with acute liver failure with mild jaundice, hypoglycaemia,

Table 1 Investigations for acute and chronic liver failure in children

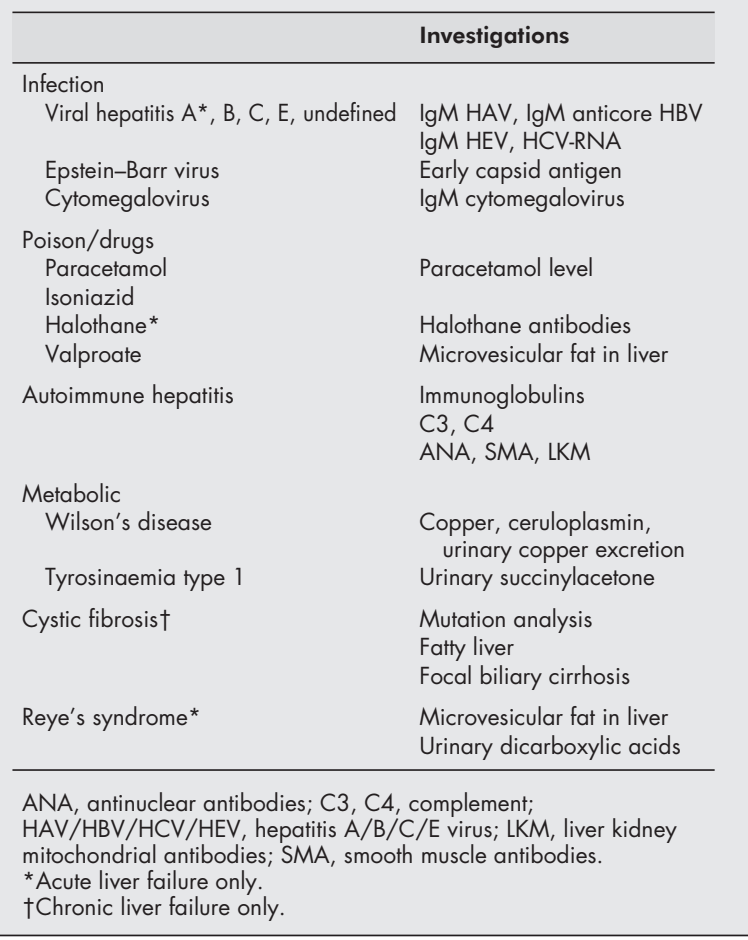

\section{Box 2: Supportive management of fulminant hepatitis}

- No sedation except for procedures

- Monitor: neurological observations 4-6 hourly, gastric pH (>5), blood glucose (>4 mmol/l), acid-base/electrolytes, prothrombin time, partial thromboplastin time.

- Fluid balance: $75 \%$ maintenance. Maintain circulating volume with colloid/fresh frozen plasma.

Consider: frusemide infusion, haemofiltration.

- Coagulation support (fresh frozen plasma, cryoprecipitate).

- Drugs: vitamin K $(2-10 \mathrm{mg})$, ranitidine $(3 \mathrm{mg} / \mathrm{kg})$, sucralfate $(2-4 \mathrm{~g} /$ day $)$, lactulose $(5-20 \mathrm{ml} /$ day $)$, $\mathrm{N}$-acetylcysteine $(70 \mathrm{mg} / \mathrm{kg} / 6$ hour $),+/$ - broad spectrum antibiotics, fluconazole.

- Nutrition: enteral feeding (1-2 g protein/day), parenteral nutrition if ventilated.

coagulopathy, encephalopathy, ascites, and occasionally hyperinsulinism. The diagnosis is based on increased plasma tyrosine, phenylalanine, methionine and confirmed by identifying a toxic metabolite, succinylacetone in the urine, or by enzyme analysis of skin fibroblasts. Management includes supportive management of acute liver failure (box 2) and with 2 (2 nitro-4-trifluoromethylbenzoyl)-1,3-cyclohexenedione (NTBC), ${ }^{5}$ which prevents the formation of toxic metabolites and allows hepatic regeneration. Children who fail to respond to NTBC should be offered liver transplantation. ${ }^{6}$

\section{Mitochondrial disorders}

Mitochondrial disorders present with acute liver failure in the context of multiorgan disease. There are many different clinical phenotypes and modes of inheritance including transmission through maternal DNA. The disorders include deficiencies of the electron transport chain enzymes or depletion of mitochondrial DNA. Neonates and infants present with jaundice, coagulopathy, and neurological features very similar to hepatic encephalopathy and multiorgan failure (cardiac, renal, bone marrow). Infants have metabolic acidosis with a raised blood lactate. Other useful investigations are an increased plasma 3-hydroxybutyrate-acetoacetate ratio $(>2)$ or detection of abnormal organic acids such as urinary 3-methylglutaconic acid. Evidence of multiorgan involvement is confirmed by muscle biopsy which may demonstrate abnormal mitochondria while an increased cerebrospinal fluid lactate, or the presence of cerebral atrophy on computed tomography or magnetic resonance imaging, suggests neurological involvement. Liver histology demonstrates microvesicular fatty infiltration, hepatocyte degeneration, and micronodular cirrhosis. The diseases are fatal and liver transplantation is not indicated because of progressive neurological disease and multiorgan failure. ${ }^{7}$

\section{Familial haemophagocytic syndrome}

Haemophagocytic syndrome is probably an autosomal recessive disorder due to a defect in immunomodulation, but may also be virally induced. Infants present with multiorgan failure with jaundice, hepatosplenomegaly, fever, skin rash, and pancytopenia. The diagnosis is confirmed by finding erythrophagocytosis in bone marrow, liver, and occasionally

\section{Box 3: Management of neonatal haemochromatosis}

- N-acetylcysteine (140 mg/kg; 70 mg/kg 6 hour).

- Prostaglandin El $(0.4-0.6 \mu \mathrm{g} / \mathrm{kg} / \mathrm{hour})$.

- Desferrioxamine (30 mg/kg/day).

- Selenium (2-3 $\mu \mathrm{g} / \mathrm{kg} /$ day).

- Alpha-tocopherol polyethylene glycol succinate (20-30 $\mathrm{IU} / \mathrm{kg} /$ day). 
cerebrospinal fluid. Confirmatory investigations include elevated plasma triglycerides and increased serum ferritin. Treatment includes supportive management of acute liver failure, etoposide, and corticosteroids. Cyclosporin and anti-T lymphocyte globulin have also been used with some effect in children who have achieved remission. Liver transplantation is not indicated but bone marrow transplantation is a possibility. ${ }^{8}$

\section{ACUTE LIVER FAILURE IN OLDER CHILDREN Viral hepatitis}

Acute hepatitis A is the commonest cause in childhood, and has the best prognosis. Hepatitis B, C, and D rarely cause fulminant hepatic failure in childhood while hepatitis $\mathrm{E}$ virus may be associated with fulminant hepatic failure in children returning from the Indian subcontinent. Virus $G$ and transfusion transmitted virus, which are parenterally transmitted viruses, have not been proved to cause liver disease. Hepatitis secondary to other viruses such as Epstein-Barr virus and parvovirus B19 occasionally lead to fulminant hepatitis. Approximately $50 \%$ of children with viral fulminant hepatic failure will have no obvious aetiology and are classified as non-A-G fulminant hepatitis. The prognosis for this group is poor and few recover without liver transplantation. ${ }^{1}$ The clinical presentation includes a prodromal illness with anorexia, vomiting, lethargy, gradual onset of jaundice, coagulopathy, and encephalopathy. The diagnosis is made by measuring viral serology and excluding other causes of fulminant hepatitis (table 1).

\section{Autoimmune hepatitis}

Autoimmune hepatitis (type I and type II) occasionally presents with acute hepatic failure, although fulminant hepatitis is more common in type II and chronic liver failure with type I. The clinical presentation is similar to viral hepatitis but there is often a history of recurrent jaundice with lethargy, fatigue, and weight loss. ${ }^{9}$

The diagnosis is confirmed by identifying elevated immunoglobulins particularly IgG, reduced levels of complement (C3, C4) and non-specific autoantibodies (type I: antinuclear antibody and antismooth muscle; type II: antiliver kidney microsomal antibodies). Therapy includes supportive management and initiating immunosuppression with prednisolone $(2 \mathrm{mg} / \mathrm{kg})$. Steroids should be limited to a maximum dose of $60 \mathrm{mg}$ as they may exacerbate encephalopathy. Liver transplantation is indicated for those children who do not respond to immunosuppression.

\section{Drug induced liver failure}

A number of drugs and toxins are associated with fulminant hepatic failure in children, but in Britain, paracetamol overdose in adolescent girls is the commonest cause of liver toxicity with doses of greater than $150 \mathrm{mg} / \mathrm{kg}$. Maximal liver injury develops between 2-4 days after the overdose and may be associated with metabolic acidosis and renal failure. The risk of significant acute liver failure is increased if other drugs-for example, anticonvulsant therapy, recreational drugs such as ecstasy or alcohol, are ingested. Management includes estimation of serum paracetamol levels, and prompt treatment with intravenous $N$-acetylcysteine to prevent massive hepatic necrosis (box 2).

The risk of acute liver failure with sodium valproate is particularly high in the first three years of life but may occur at any age including young adults. It may be the first presentation of an underlying mitochondrial disease. The presentation is usually with jaundice, vomiting, increased frequency of convulsions, oedema, and encephalopathy. Treatment is supportive. Liver transplantation is contraindicated because of the underlying metabolic disorder or the presence of multiorgan disease. ${ }^{10}$
Anticonvulsant medication with carbamazepine usually produces a cholestatic hepatitis but may rarely cause fulminant hepatitis.

\section{Metabolic liver disease}

Wilson's disease is the commonest metabolic cause of fulminant hepatic failure in children over the age of 3. The presentation is variable and may mimic viral hepatitis. The diagnosis is suggested by finding haemolysis on a blood film, a relatively low alkaline phosphatase $(<600$ IU/l), raised urinary copper (before and after penicillamine challenge), a low serum copper and caeruloplasmin. Kayser-Fleischer rings may not be obvious but there may be a response to D-penicillamine $(20 \mathrm{mg} / \mathrm{kg}$ per day). Liver transplantation is indicated for those who do not respond or have advanced liver failure with severe coagulopathy and encephalopathy. ${ }^{11}$

\section{Diagnosis}

The diagnosis of acute liver failure is established by performing standard liver function tests and coagulation. Investigations will show a marked conjugated hyperbilirubinaemia, raised aminotransferases $(>10000 \mathrm{IU} / \mathrm{l})$, raised plasma ammonia (>100 IU/l), and coagulopathy (prothrombin time $>40$ seconds). Liver biopsy is contraindicated because of abnormal coagulation but can be performed by the transjugular route if essential for diagnosis. Specific investigations for infection, drugs, and metabolic disorders are in table 1.

\section{Management}

Management of acute liver failure requires intensive support and referral to a specialist paediatric liver unit for early consideration for liver transplantation. The essential aspects are:

- To prevent complications such as encephalopathy and cerebral oedema, sepsis, gastrointestinal bleeding, renal failure, and multiorgan failure.

- To assess prognosis and consider liver transplantation.

- To provide hepatic support.

Essential management includes:

- Fluid restriction to $75 \%$ of maintenance to prevent cerebral oedema and reduce encephalopathy.

- Maintain glucose levels $>4.0 \mathrm{mmol} / \mathrm{l}$.

- Prevent gastrointestinal bleeding with ranitidine $3 \mathrm{mg} / \mathrm{kg}$ and sucralfate $2-4 \mathrm{~g}$ /day.

- Prevent sepsis using broad spectrum antibiotics and antifungal agents.

- Control coagulopathy with intravenous vitamin K (2-10 $\mathrm{mg}$ ); treatment of severe coagulopathy (prothrombin time $>60$ seconds) with fresh frozen plasma and cryoprecipitate.

Treatment of cerebral oedema is difficult. Fluid restriction and intravenous mannitol ( $0.5 \mathrm{~g} / \mathrm{kg}$ over $4-6$ hours $)$ is useful term but elective ventilation is required if grade II or III hepatic coma develops. Monitoring the intracranial pressure, using intracranial electrodes may improve selection for liver transplantation but does not affect survival.

Renal failure with oliguria and anuria should be managed by maintaining circulating volume with colloid or fresh frozen plasma. Intravenous frusemide or a frusemide infusion may be considered but haemofiltration may be necessary to control cerebral oedema.

Artificial liver support using porcine hepatocytes or hepatoma cell lines have improved coagulopathy and reduced encephalopathy in both adults and children. Although useful as a "bridge to transplantation", long term outcome and survival were not affected. ${ }^{12}$

\section{Selection for liver transplantation}

Prognostic factors for survival with acute liver failure are not well established in children. In general children with 
Box 4: Aetiology of chronic liver failure in children

Cholestatic liver disease

- Biliary atresia.

- Idiopathic neonatal hepatitis.

- Alagille's syndrome.

- Familial intrahepatic cholestasis.

Metabolic liver disease

- Alpha-1-antitrypsin deficiency.

- Tyrosinaemia type I.

- Wilson's disease.

- Cystic fibrosis.

- Glycogen storage type IV.

Chronic hepatitis

- Autoimmune +/- sclerosing cholangitis.

- Postviral (hepatitis B, C, other).

- Fibropolycystic liver disease +/- Caroli's syndrome.

- Primary immunodeficiency.

- Non-alcoholic steatohepatitis.

metabolic liver disease or severe coagulopathy (for example, neonatal haemochromatosis) are unlikely to recover ${ }^{13}$ while children with paracetamol poisoning or hepatitis A have the best prognosis for a spontaneous recovery.

A poor prognosis and immediate referral for liver transplantation is indicated by:

- Prothrombin time $>60$ seconds.

- Decreasing transaminase levels.

- Rising bilirubin >300 mmol/l.

- Decreasing liver size.

- Acid-base $\mathrm{pH}<7.3$.

- Hypoglycaemia $<4 \mathrm{mmol} / \mathrm{l}$ with increasing dextrose requirement.

- Hepatic coma grade 2 or 3.

The presence of irreversible multisystem disease, such as mitochondrial disorders and erythophagocytosis, or obvious cerebral damage on computed tomography or magnetic resonance imaging are contraindications for liver transplantation.

\section{CHRONIC LIVER FAILURE}

\section{Cholestatic neonatal liver disease}

Biliary atresia remains the commonest cause of liver failure in children worldwide and is the main indication for liver transplantation (box 4). ${ }^{14}$ It is a disease of unknown aetiology in which there is destruction of the extrahepatic and intrahepatic biliary ducts leading to cholestasis, fibrosis, and cirrhosis. The clinical features include progressive obstructive jaundice, and failure to thrive. The diagnosis is based on evidence of biliary obstruction and liver histology which demonstrates fibrosis, cholestasis, and proliferation of biliary ductules.

Surgical removal of the fibrosed biliary tree and formation of a Roux-en-Y anastomosis (Kasai portoenterostomy) is a palliative procedure, which achieves biliary drainage in $60 \%$ of infants. Medical management consists of prevention of cholangitis with low dose oral antibiotics (for example, amoxycillin, $125 \mathrm{mg} /$ day; cephalosporin $125 \mathrm{mg} /$ day; or trimethoprim $120 \mathrm{mg} /$ day), and nutritional and family support. Recurrent cholangitis, cirrhosis, and portal hypertension are inevitable despite good biliary drainage and are less likely in experienced paediatric units, which is now government policy. ${ }^{15}$ If surgery is unsuccessful, liver transplantation is an immediate requirement.

\section{Alpha-1-antitrypsin deficiency}

Alpha-1-antitrypsin deficiency is the commonest inherited disorder to present in the neonatal period. Infants present with cholestasis, failure to thrive, hepatomegaly, or a vitamin $\mathrm{K}$ responsive coagulopathy. Differentiation from biliary atresia may be difficult, but the diagnosis is confirmed by detection of a low level of alpha-l-antitrypsin $(<0.9 \mathrm{~g} / \mathrm{l})$ with phenotype protease inhibitor (PIZZ or PISZ). Liver histology demonstrates giant cell hepatitis with granules of alpha-1antitrypsin in the hepatocytes. The outcome is variable. Jaundice diminishes in most babies and 30\% recover; however, 30\% develop inactive fibrosis or cirrhosis and $40 \%$ develop chronic liver failure requiring transplantation in childhood. Management is supportive and varies with clinical course. ${ }^{16}$

\section{Cystic fibrosis}

Cystic fibrosis may present with cholestasis, respiratory symptoms and failure to thrive in infancy and should be confirmed by a sweat test at 6 weeks of age or by mutation analysis.

\section{Biliary hypoplasia}

There are a number of cholestatic disorders which are associated with intrahepatic biliary hypoplasia-that is, an absence or reduction in the number of interlobular bile ducts or ductules associated with a normal sized portal vein and hepatic arteries. Recent advances in molecular genetics have defined these disorders and provided information about function. ${ }^{17}{ }^{18}$

Alagille's syndrome is the best known of these disorders. It is an autosomal dominant condition with an incidence of $1 / 100000$ live births. The genetic defect is on the jagged 1 gene on chromosome 20. It is a multisystem disorder with cardiac, facial, renal, ocular, and skeletal abnormalities. Infant have persistent cholestasis, severe pruritis, hepatomegaly, and a failure to thrive. Prognosis depends on the severity of liver and cardiac disease. At least $50 \%$ of children will regain normal liver function by adolescence. Liver transplantation is indicated if there is progression to cirrhosis and portal hypertension, or intractable pruritis and poor quality of life.

Familial intrahepatic cholestasis (FIC) has now been defined as three separate disorders with defects on chromosome 2 , and 18 (FICl, 2 , and 3), ${ }^{19}$ which lead to abnormalities of bile salt transport. Children present with cholestasis, pruritis, and with a low serum glutamyltranspeptidase in FIC 1 and 2 and a high gamma-glutamyltransferase in FIC 3. Intensive nutritional support and treatment of pruritis is essential. Surgical biliary diversion may relieve pruritis, but liver transplantation is currently the treatment of choice for liver failure.

\section{METABOLIC DISORDERS}

\section{Tyrosinaemia type I}

Tyrosinaemia may present with acute liver failure in the neonate (see above) or with chronic liver disease in the older child. Treatment of chronic tyrosinaemia includes a low protein diet to reduce tyrosine levels, additional vitamin D to prevent rickets, and NTBC, which prevents the breakdown of tyrosine and reverses the clinical and biochemical complications. Liver transplantation is now only indicated for children who fail to respond to NTBC or with suspicion of hepatic malignancy (a rising alpha-fetoprotein, increasing nodularity on hepatic ultrasound, computed tomography, or magnetic resonance imaging). ${ }^{56}$

\section{Cystic fibrosis}

As long term survival improves in children with cystic fibrosis, liver transplantation has become a viable alternative and this disease is now the commonest indication in older children. Management is supportive and involves nutritional therapy, particularly vitamin A and E supplementation and ursodeoxycholic acid $(20 \mathrm{mg} / \mathrm{kg}) .{ }^{20}$ Hepatic decompensation is a late feature of cystic fibrosis liver disease, but portal hypertension is common and bleeding oesophageal varices may be a serious recurrent problem, which requires standard therapy. Selection 
for liver transplantation is indicated only for those children with hepatic decompensation (falling serum albumin, prolonged coagulation unresponsive to vitamin K), severe malnutrition, intractable complications of portal hypertension unresponsive to medical management, ascites, uncontrolled variceal bleeding. Careful assessment of pulmonary function is required as severe lung disease ( $<60 \%$ of lung function) may indicate the necessity for a heart, lung, and liver transplant. $^{21}$

\section{Wilson's disease}

Wilson's disease is an autosomal recessive disorder with an incidence of five per million live births. Copper accumulates in the liver, brain, kidney, and cornea. The condition seldom presents before 3 years of age, but any form of acute (see above) or chronic liver disease is possible. Neurological features tend to occur in adolescence, while Kayser-Fleischer rings may be visible in children over 7 years of age.

Management is with penicillamine, $20 \mathrm{mg} / \mathrm{kg} / \mathrm{day}$, in combination with oral zinc and pyridoxine $50 \mathrm{mg} /$ day, which acts by chelating copper, which is then excreted in the urine. Untreated, $30 \%$ of children die. Liver transplantation is indicated if the liver failure is unresponsive to penicillamine. ${ }^{11}$

\section{Chronic hepatitis}

Autoimmune liver disease is the commonest liver disease in older children, but is an unusual cause of liver failure because the majority of children with either autoimmune liver disease type I or type II respond to immunosuppression with prednisolone $2 \mathrm{mg} / \mathrm{kg}$ or azathioprine $(0.5-1 \mathrm{mg} / \mathrm{kg}$ ). Liver transplantation is indicated for those children who do not respond to immunosuppression despite the use of second line drugs such as cyclosporin A or tacrolimus or those who present with fulminant hepatic failure. ${ }^{9}$

Most children with chronic hepatitis B or C are asymptomatic carriers in whom the development of cirrhosis, portal hypertension and/or hepatocellular carcinoma may evolve over 20-30 years, and rarely requires treatment in childhood.

\section{Non-alcoholic steatohepatitis}

The increase in childhood obesity and the recognition of insulin resistance in a number of inherited disorders has lead to the diagnosis of this disorder in childhood. The long term outcome is not determined, but there may be a response to weight reduction. Therapeutic trials with metformin and other drugs have not been evaluated in childhood. ${ }^{22}$

\section{Cirrhosis}

Cirrhosis and portal hypertension is the end point for all forms of progressive chronic liver disease. It may be compensated or decompensated. Hepatic decompensation occurs when there is loss of hepatic synthetic function and the development of complications such as malnutrition, bleeding oesophageal varices, ascites, encephalopathy, and hepatorenal failure (box 5).

The clinical features of decompensated cirrhosis include palmar and plantar erythema, telangiectasia, malnutrition, hypotonia, and hepatosplenomegaly with ascites. Jaundice may be absent.

\section{Box 5: Complications of cirrhosis in children}

- Malnutrition and growth failure.

- Coagulopathy.

- Portal hypertension: hypersplenism, variceal bleeding ascites, encephalopathy.

- Hepatopulmonary syndrome.

- Hepatorenal syndrome.

- Bacterial infections, spontaneous bacterial peritonitis.

- Hepatocellular carcinoma (rare).

\section{Box 6: Management of chronic liver failure}

Nutritional support

- Energy intake 120\%-150\% (recommended daily amount).

- Carbohydrate $15-20 \mathrm{~g} / \mathrm{kg} /$ day.

- Protein 3-4 g/kg/day.

- Fat $8 \mathrm{~g} / \mathrm{kg} /$ day $(50 \%$ medium chain triglyceride)

- Fat soluble vitamins.

Fluid balance

- Avoid excess sodium (<2 mmol/ $\mathrm{kg}$ ).

- Ascites: spironolactone (3 $\mathrm{mg} / \mathrm{kg})$, frusemide $(0.5-2$ $\mathrm{mg} / \mathrm{kg}$ ), albumin infusion, paracentesis.

\section{Encephalopathy}

- Low protein $(2 \mathrm{~g} / \mathrm{kg})$.

- Lactulose (5-20 ml/day).

\section{Coagulopathy}

- Vitamin K (2 - $10 \mathrm{mg} /$ day).

- Fresh frozen plasma, cryoprecipitate, platelets.

Diagnosis-although cirrhosis is a histological diagnosis (extensive fibrosis and regenerative nodules), it is generally associated with:

- An echogenic liver with splenomegaly and varices on ultrasonography.

- Oesophageal and gastric varices on endoscopy.

- Mild transaminitis (aspartate aminotransferase and alanine aminotransferase $>3 \times$ normal).

- Increased serum alkaline phosphatase and gammaglutamyltransferase (twice normal).

- Low serum albumin $(<30 \mathrm{~g} / \mathrm{l})$.

- Low serum calcium and phosphate secondary to rickets.

- Anaemia.

- Prothrombin time $>20$ seconds.

- Slow irregular, low frequency waves on electroencephalography.

MANAGEMENT OF CHRONIC LIVER FAILURE (BOX 6) The aim of management of chronic liver failure is to:

- Prevent progression of liver failure with specific medication (see above).

- Provide nutritional support to prevent malnutrition.

- Control or prevent complications.

Sufficient calories to prevent malnutrition and to overcome fat malabsorption and ongoing catabolism can be provided by a high calorie, protein feed $(110 \%-160 \%$ of the recommended daily amount) with $50 \%$ medium chain triglycerides (box 6). Nocturnal nasogastric enteral feeding may be necessary to provide the volume required and parents can be taught to do this at home. It may be difficult to achieve this intake in fluid restricted children, thus a modular concentrated feed may be appropriate for young babies, while calorie supplements are more suitable for older children. If enteral feeding is not tolerated, due to ascites, variceal bleeding or recurrent hepatic complications, parenteral nutrition in normal amounts is required.

Generous oral supplements of the fat soluble vitamins are essential especially in cholestatic children (box 6) and should include:

- Vitamin A, 5-15 000 IU/day.

- Vitamin D (alphacalcidol), $50 \mathrm{ng} / \mathrm{kg} /$ day.

- Vitamin E, 50-200 mg/day.

- Vitamin K, 2.5-5 mg/day. 
Pruritis is difficult to treat, and may be intractable in children with biliary hypoplasia. Therapy with more than one drug is usually required. These include: cholestyramine, 1-2 g/day, phenobarbitone, 5-15 mg/kg/day, ursodeoxycholic acid 20 $\mathrm{mg} / \mathrm{kg}$ and rifampicin $50 \mathrm{mg} / \mathrm{kg} / \mathrm{day}$, ondansetron 2-4 $\mathrm{mg} / \mathrm{twice}$ a day, naltrexone $6-20 \mathrm{mg} /$ day.

Metabolic bone disease may be severe with pathological fractures. Treatment with infusions of bisphonates are currently under evaluation. ${ }^{23}$

\section{OESOPHAGEAL VARICES}

Oesophageal or rectal varices develop inevitably with increasing hepatic fibrosis and portal hypertension. Prophylactic therapy is not recommended.

\section{MANAGEMENT OF VARICEAL HAEMORRHAGE}

The basic principles are: to assess the site and extent of the bleed, to resuscitate with albumin, fresh frozen plasma, red cell transfusions, $\mathrm{H}_{2}$ blockers such as ranitidine, or proton pump inhibitors such as omeprazole are also used, although the efficacy is unknown.

Acute bleeding required urgent therapy with intravenous octreotide (3-5 $\mu \mathrm{g} / \mathrm{kg} / \mathrm{hour}$ ) and/or glypressin (0.3-1 unit $/ \mathrm{kg} /$ hour) or vasopressin $(0.2-0.4$ units $/ \mathrm{kg} /$ hour $)$ in order to reduce portal pressure.

Once the child has been stabilised, it is essential to confirm the diagnosis by endoscopy and to carry out therapeutic sclerotherapy or band ligation as required. If bleeding continues despite sclerotherapy or band ligation, balloon tamponade with the modified Sengstaken-Blakemore tube may be effective (fig l).

There are now a number of large series reporting the results of endoscopic sclerotherapy, ${ }^{24}{ }^{25}$ demonstrating successful variceal ablation from $84 \%-96 \%$ with rebleeding rates of $4 \%-45 \%$ (box 1). There are significant complications which include oesophageal ulceration $(20 \%)$, stricture $(16 \%)$, and recurrent varices $(8 \%)$. Endoscopic sclerotherapy is effective long term in obliterating varices but is not effective for gastric varices or hypersplenism.

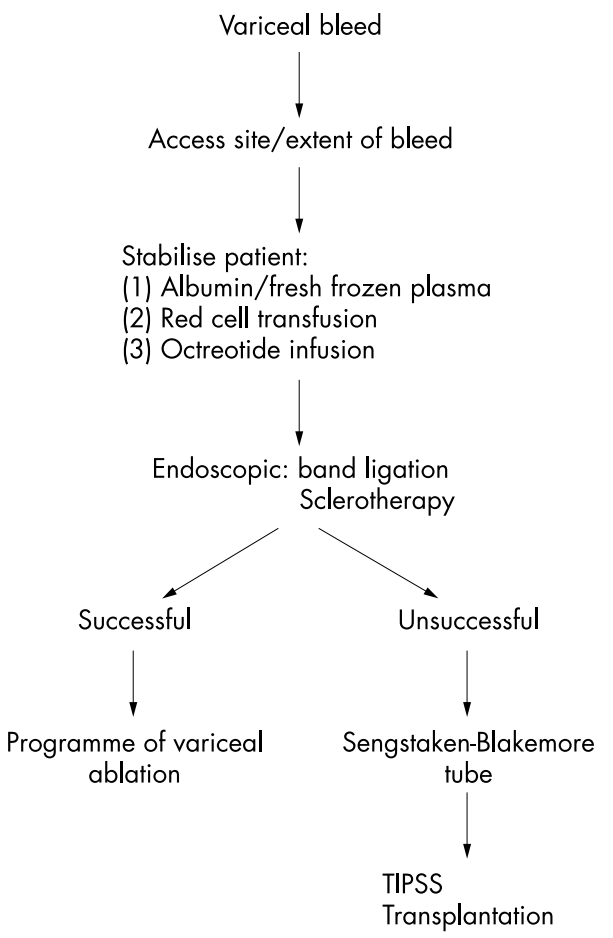

Figure 1 Management of acute variceal bleed in childhood.
Injection sclerotherapy has largely been replaced by band ligation. ${ }^{26}$ There are few reported studies in children but preliminary results indicate that between $70 \%-100 \%$ of varices are ablated with rebleeding rates of $15 \%-30 \%$ and a lower complication rate, although dysphagia after therapy is more common.

In a small number of children with intractable variceal bleeding, haemorrhage may be controlled by insertion of a transjugular intrahepatic portosystemic stent shunt (TIPSS). ${ }^{27}$ This radiological technique involves identification of the anatomy of the portal vein by hepatic and mesenteric angiography, cannulation of the right internal jugular vein, and introduction of the cannula and biopsy needle into the right hepatic vein. A guidewire is passed through a needle into the main portal vein and a stent (usually a metal prosthesis) is placed between the hepatic vein and the main portal vein. A number of small studies reporting the use of TIPSS in children have indicated success rates of $80 \%-100 \%$. Complications include occlusion of the stent, infection, and development of encephalopathy.

The use of TIPSS to control portal hypertension in children with compensated liver disease such as cystic fibrosis is an increasing indication for this procedure. ${ }^{26}$

The use of propranolol as prophylactic therapy remains unproved in children. ${ }^{28}$

\section{SEPSIS}

Sepsis, particularly ascending cholangitis and spontaneous bacterial peritonitis, is common and may precipitate encephalopathy or acute on chronic liver failure. Treatment with appropriate broad spectrum antibiotics, such as cefuroxime (20 mg/kg/dose three times a day); amoxil $(25 \mathrm{mg} / \mathrm{kg} /$ dose three times a day), and metronidazole $(8 \mathrm{mg} / \mathrm{kg} / \mathrm{dose}$ three times a day) are useful first line drugs until bacterial cultures are positive.

Salt and water retention leading to ascites and cardiac failure should be effectively managed with diuretics and salt and water restriction (box 6). It is essential to consider intervention with haemodialysis and/or haemofiltration if acute renal failure or hepatorenal failure develop. Haemodialysis is rarely required in chronic liver failure unless there is acute decompensation.

Chronic encephalopathy is difficult to detect in children, but may present with regression in school work, lack of energy, and drowsiness. It is best treated with lactulose, although protein restriction $(2 \mathrm{~g} / \mathrm{kg})$ may be necessary

Coagulation support with vitamin $\mathrm{K}$, fresh frozen plasma, cryoprecipate, and platelet transfusions (for hypersplenism) are needed to cover procedures. Hypersplenism rarely requires treatment in itself.

\section{LIVER SUPPORT}

Recent trials in adults have evaluated a modified dialysis method using a molecular absorbent recirculating system, which may improve short term survival in end stage liver failure. Trials have not yet been performed in children. ${ }^{29}$

\section{TIMING OF TRANSPLANTATION FOR CHILDREN WITH CHRONIC LIVER FAILURE}

As many children with cirrhosis and portal hypertension have well compensated liver function it may be difficult to decide when to transplant. Useful indicators include:

- Persistent rise in total bilirubin $>150 \mu \mathrm{mol} / \mathrm{l}$.

- Prolongation of prothrombin ration (international normalised ratio > 1.4).

- Fall in serum albumin $<35 \mathrm{~g} / \mathrm{l}$.

- Reduction in psychosocial development.

- Failure to thrive despite nutritional intervention. 


\section{Key references}

- Gregorio GV, Portmann B, Karani J, et al. Autoimmune hepatitis/sclerosing cholangitis overlap syndrome in childhood: a 16-year prospective study. Hepatology 2001;33: 544-53.

- McKiernan PJ, Baker AJ, Kelly DA. The frequency and outcome of biliary atresia in the UK and Ireland. Lancet 2000;355:25-9.

- Thompson R, Strautnieks S. Inherited disorders of transport in the liver. Curr Opin Genet Dev 2000;10:310-3.

- Rashid M, Roberts E. Non-alcoholic steatohepatitis in children. J Pediatr Gastroenterol Nutr 2000;30:48-53

- Van Mourik IDM. Long term nutrition and neurodevelopmental outcome of liver transplantation in infants aged less than 12 months. J Pediatr Gastroenterol Nutr 2000;30 269-76.

- Chronic hepatic encephalopathy.

- Refractory ascites.

- Recurrent variceal bleeding despite optimum management.

- Intractable pruritis.

- Severe metabolic bone disease.

- Diminishing quality of life.

It is essential that children with chronic liver disease should be referred for transplantation before the complications of their liver disease adversely impairs the quality of their lives and before growth and development are retarded. ${ }^{30}$

Assessment should be performed at a specialist paediatric liver unit. Preparation includes intensive nutritional support, management of hepatic complications, and education and counselling of the child and family. Irreversible extrahepatic disease is the only absolute contraindication to transplantation. As live vaccines are contraindicated in the immunosuppressed child, it is important to ensure that routine immunisations are complete. Ideally hepatitis $\mathrm{A}$ and $\mathrm{B}$ vaccination should be prescribed before transplant.

The most important aspect of the transplant assessment is counselling and preparation of the child and family. A skilled multidisciplinary team, including play therapist and psychologist, is essential to the success of this preparation. Psychological preparation in children older than 2 years may be successfully achieved through innovative play therapy.

\section{LIVER TRANSPLANTATION}

Early consideration for liver transplantation is necessary to expedite the search for a donor liver. Organs are matched by blood group and size. Age and size are no longer contraindications for liver transplantation but due to the shortage of size matched organs, most children will receive either a reduced or split liver graft. Auxiliary liver transplantation (in which part of the recipient liver is left to regenerate in situ) is controversial treatment for fulminant hepatic failure but has the potential for the graft to be removed if the original liver regenerates. ${ }^{31}$ It is not suitable for transplantation for children with metabolic liver disease or autoimmune hepatitis. Living related donation from a family member is an alternative possibility, particularly as there is a donor shortage, although this places considerable pressure on the family.

\section{Complications and outcome}

One year survival is $90 \%-95 \%$ and five year survival more than $80 \%$. Morbidity is high; sepsis (70\%), rejection (60\%), vascular thrombosis $(10 \%)$, and biliary complications $(20 \%)$ are expected, but after increased medical and surgical experience, technical problems such as primary graft non-function and vascular thrombosis are now almost non-existent, and the retransplantation rates in children have fallen. ${ }^{32}$ The difficult

\section{Practice points}

(1) Acute liver failure is a rare but fatal disease with mortality of $70 \%$ without transplantation. The commonest causes are viral hepatitis or inherited metabolic liver disease. Referral to a specialist unit, supportive management, and consideration for liver transplantation is essential.

(2) The commonest causes of chronic liver failure are extrahepatic biliary atresia or inherited metabolic liver disease in neonates, and auto-immune hepatitis and cystic fibrosis in older children. Nutritional support and management of hepatic complications have improved short term outcome.

(3) Liver transplantation for acute or chronic liver failure achieves good quality life in over $70 \%$ of children.

\section{Further information}

- Children's Liver Disease Foundation, 35-37 Great Charles Queensway, Birmingham B3 3JY, UK (tel: 0121212 3839, fax: 0121212 4300; www.childliverdisease.org)

- Department of Health, NSCAG: www.doh.gov.uk/nscag

- Paediatric hepatology: www.pedihepa.org

- Gastroenterology: www.gastrohep.com

problem of acute and chronic rejection, which historically is less common in children than in adults has improved after advances in immunosuppression with drugs which are more easily absorbed such as cyclosporin microemulsion (Neoral) ${ }^{33}$ or more potent such as tacrolimus which has reduced the incidence of chronic rejection. ${ }^{34}$

As long term survival increases, attention has now focused on the quality of life achieved by children undergoing transplantation. Long term studies in children and adolescents have demonstrated the striking nutritional rehabilitation achieved after successful liver transplantation. ${ }^{35}$

More importantly there is clear maintenance of psychosocial development both physically and intellectually and it is expected that children will return to a normal life attending school and college. Many studies have demonstrated normal, if delayed, pubertal development and growth spurts have been clearly documented and successful pregnancies reported. ${ }^{36}$

The long term effects of immunosuppression remain the greatest challenge. Renal dysfunction occurs in approximately $30 \%$, while the incidence of post-transplant lymphoproliferative disease and other tumours is approximately 5\%-6\%. Long term data indicate that some diseases such as hepatitis B and $\mathrm{C}$ and autoimmune hepatitis, will recur after liver transplantation. Despite this, the successful development of liver transplantation has revolutionised the outlook for many children dying of acute or chronic liver failure, and leads to good quality life.

\section{QUESTIONS (ANSWERS AT END OF PAPER)}

\section{Indications for liver transplantation include:}

(A) Failed Kasai portoenterostomy for biliary atresia

(B) Thalassaemia major with hepatic involvement

(C) Alpha-1-antitrypsin disease leading to cirrhosis

(D) Localised hepatic tumour

(E) Fulminant hepatitis (non-A-G)

\section{Absolute contraindications for liver transplantation} include:

(A) A patent portal vein

(B) Weight less than $10 \mathrm{~kg}$ at operation 
(C) Severe hepatic encephalopathy

(D) Herniation of brain stem

(E) Untreatable congenital heart disease

\section{Wilson's disease:}

(A) Is a disorder of iron metabolism

(B) May respond to penicillamine therapy

(C) Is associated with haemolysis

(D) Rarely causes acute liver failure

(E) Recurs after liver transplantation

\section{Neonatal liver disease:}

(A) Is associated with breast feeding

(B) May lead to severe malnutrition

(C) Pruritus is an important symptom

(D) Never requires liver transplantation

(E) Fat soluble vitamins are usual therapy

\section{Cystic fibrosis}

(A) Is an important indication for liver transplantation

(B) Lung and liver disease rarely occur together

(C) Portal hypertension is a common complication

(D) Therapy includes ursodeoxycholic acid

(E) Liver disease may recur after transplantation

\section{REFERENCES}

1 Alonso EM, Superina RA, Whitington PF. Fulminant hepatitis and acute liver failure. In: Kelly DA, ed. Diseases of the liver and biliary system in children. Oxford: Blackwell Science, 1999: 77-96.

2 Beath SV, Boxall EH, Watson RM, et al. Fulminant hepatitis B in infants born to anti-Hbe hepatitis B carrier mothers. BM 1992;304:1 169-70.

3 Friedt M, Gerner P, Lausch E, et al. Mutations in the basic core promoto and the precore region of hepatitis $B$ virus and their selection in children with fulminant and chronic hepatitis B. Hepatology 1999;29:1252-8.

4 Sigurdsson L, Reyes J, Kocoshis SA, et al. Neonatal hemochromatosis: outcome of pharmacologic and surgical techniques. J Pediatr Gastroenterol Nutr 1998;26:85-9

5 Lindstetd S, Holme E, Lock EA, et al. Treatment of hereditary tyrosinaemia type I by inhibition of 4-hydroxyphenylpyruvate dioxygenase. Lancet 1992;340:813-7.

6 Mohan N, McKiernan P, Preece MA, et al. Indications and outcome of liver transplantation in tyrosinemia type 1. Eur J Pediatr 1999; 158:S49-54

7 Thomson M, McKiernan P, Buckels J, et al. Generalised mitochondrial cytopathy is an absolute contraindication to orthotopic liver transplantation in childhood. I Pediatr Gastroenterol Nutr 1998;26:478-81.

8 Jabado N, de Graeff-Meeder ER, Cavazzana-Calvo M, et al. Treatmen of familial hemophagocytic lymphohistiocytosis with bone marrow transplantation from HLA genetically nonidentical donors. Blood 1997:90:4743-8.

9 Gregorio GV, Portmann B, Karani J, et al. Autoimmune hepatitis/sclerosing cholangitis overlap syndrome in childhood: a 16-year prospective study. Hepatology $2001 ; 33: 544-53$.

10 Kelly DA. Liver transplantation: to do or not to do? Pediatric Transplantation 2000;4:170-2.

11 Nazer H, Ede RJ, Mowat AP, et al. Wilson's disease: clinical presentation and use of prognostic index. Gut 1986;27:1377-81.

12 Ellis AJ, Hughes RD, Wendon JA, et al. Pilot-controlled trial of the extracorporeal liver assist device in acute liver failure. Hepatology 1996;6:1557.
13 Bhaduri BR, Mieli-Vergani G. Fulminant hepatic failure: pediatric aspects. Semin Liver Dis 1996;16:349-55.

14 European Liver Transplant Registry, 2001 (http://www.eltr.org)

15 McKiernan PJ, Baker AJ, Kelly DA. The frequency and outcome of biliary atresia in the UK and Ireland. Lancet $2000 \cdot 355 \cdot 25-9$.

16 Primhak RA, Tanner MS. Alpha-1 antitrypsin deficiency. Arch Dis Child 2001;85:2-5

17 Bull LN, Carlton VE, Stricker NL, et al. Genetic and morphological findings in progressive familial intrahepatic cholestasis (Byler disease [PFIC-1] and Byler syndrome): evidence for heterogeneity. Hepatology 1997:26:155-64.

18 Thompson R, Strautnieks S. Inherited disorders of transport in the liver. Curr Opin Genet Dev 2000;10:310-3

19 Carleton VC, Knisely AS, Freimer NB. Mapping of a locus for progressive familial intrahepatic cholestasis (Byler disease) to 18q21-q22, the benign recurrent intrahepatic cholestasis region. Hum Mol Genet 1995:4:1049-53.

20 Lindblad A, Glaumann H, Srandvik B. A 2 year prospective study of the effect of ursodeoxycholic acid on urinary bile acid excretion and liver morphology in cystic fibrosis associated liver disease. Hepatology 1998;27:166-74.

21 Couetil JP, Soubrane O, Houssain DP, et al. Combined heart-lung-liver, and isolated liver transplantation for cystic fibrosis in children. Transpl Int 1997; 10:33-9.

22 Rashid $\mathbf{M}$, Roberts E. Non-alcoholic steatohepatitis in children. J Pediatr Gastroenterol Nutr 2000;30:48-53.

23 Shepherd R. Complications and management of chronic liver disease. In: Kelly DA, ed. Diseases of the liver and biliary system in children. Oxford: Blackwell Science, 1999: 189-210

24 Maksoud JG, Goncalves ME, Porta G, et al. The endoscopic and surgical management of portal hypertension in children: analysis of 124 cases. J Pediatr Surg 1991;26:178-81.

25 Paquet KJ, Lazar A. Current therapeutic strategy in bleeding oesophageal varices in babies and children and long term results of endoscopic paravariceal sclerotherapy over twenty years. Eur J Pediatr Surg 1994;4:165-72.

26 Lee WS, John P, McKiernan P, et al. Inferior vena cava occlusion and protein-losing enteropathy after liver transplantation in children. J Pediatr Gastroenterol Nutr 2002;34:413.

27 Heyman MB, LaBerge JM. Role of transjugular portosystemic shunt in the treatment of portal hypertension in pediatric patients. J Pediatr Gastroenterol Nutr 1999;29:240-9.

28 Shashidhar H, Langhans N, Grand R. Propranolol n prevension of portal hypertensive haemorrhage in children: a pilot study. J Pediatr Gastroenterol Nutr 1999;29:12-17.

29 Mitzner SR, Strange J, Klammt S, et al. Improvement of hepatorenal syndrome with extracorporeal albumin dialysis MARS: results of a prospective randomized, controlled clinical trial. Liver Transplantation 2000;6:277-86.

30 Kelly DA, Mayer AD. Liver transplantation. In: Kelly DA, ed. Diseases of the liver and biliary system in children. Oxford: Blackwell Science, 1999 293-312.

31 Van Mourik I, Melendez H, Thomson M, et al. Efficacy of neoral in the immediate post operative period in children post liver transplantation. Liver Transplant Surgery 1998;6:491-8.

32 Talbot D, Achilleos OA, Gunson BK, et al. Progress in paediatric liver transplantation: the Birmingham experience. J Pediatr Surg 1997;32:710-13.

33 Van Mourik IDM. Long term nutrition and neurodevelopmental outcome of liver transplantation in infants aged less than 12 months. J Pediatr Gastroenterol Nutr 2000;30:269-76.

34 Kelly D, Jara P, Rodeck B. Tacrolimus dual therapy versus cyclosporin-microemulsion triple therapy in paediatric liver transplantation: results from a multi-centre randomised trial. American Journal of Transplantation 2002:2:351.

35 Holt RI, Broide E, Buchanan CR, et al. Orthotopic liver transplantation reverses the adverse nutritional changes of end stage liver disease in children. Am J Clin Nutr 1997;65:534-42.

36 Carr DB, Larson AM, Schmucker BC, et al. Maternal haemodynamics and pregnancy outcome in women with prior orthotopic liver transplantation. Liver Transplantation 2000;6:213-21.

\section{ANSWERS}

1. A, C, and E are true; 2. D and E are true; 3. B and C are true; 4. B, C, and E are true; 5. A, C, and D are true. 